\title{
Supporting Information: Structural Dynamics in UV Curable Resins Resolved by In Situ 3D Printing X-ray Photon Correlation Spectroscopy
}

Benjamin M. Yavitt ${ }^{1,2}$, Lutz Wiegart'르, Daniel Salatto ${ }^{1}$, Zhixing Huang ${ }^{1}$, Maya K. Endoh ${ }^{1}$, Sascha Poeller ${ }^{3}$, Stanislas Petrash ${ }^{4, *}$, Tadanori Koga ${ }^{1, *}$

${ }^{1}$ Department of Materials Science and Chemical Engineering, Stony Brook University, Stony Brook, New York 11794-2275

${ }^{2}$ National Synchrotron Light Source II, Brookhaven National Laboratory, Upton, New York, 11973

${ }^{3}$ Adhesives Technologies, Henkel AG \& Co KGaA, 40589 Duesseldorf, Germany

${ }^{4}$ Adhesives Technologies, Henkel Corporation, Bridgewater, New Jersey, 08807

*E-mail: stan.petrash@henkel.com (S.P.), tadanori.koga@stonybrook.edu (T.K.) 
Scheme S1. Schematic overview of printing and crosslinking in LOCTITE® VP 10997-085 dual cure (UV/Thermal) thermoset nanocomposite resin.

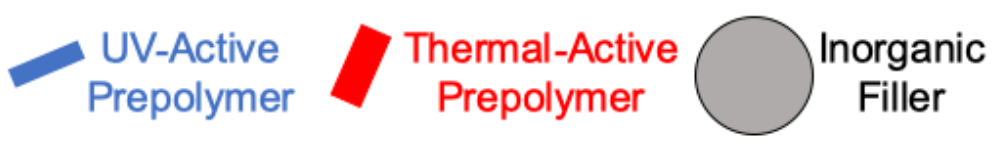

\section{Printing}

- Prepolymer Resins

- Shear Thinning
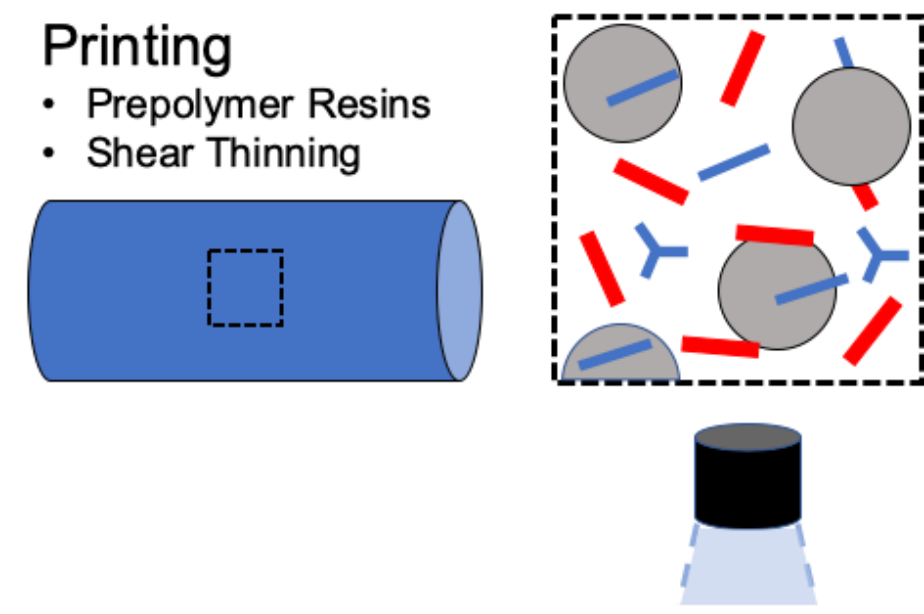

\section{In-situ UV Cure}

- Partial Crosslink

- Elastic Filament
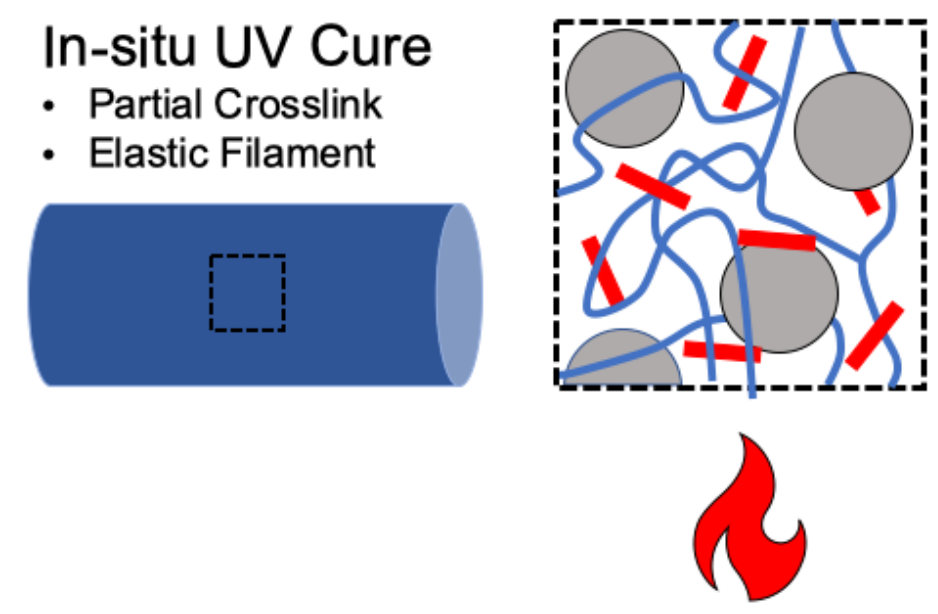

\section{Post Thermal Cure}

- Full Crosslink

- Tough Thermoset
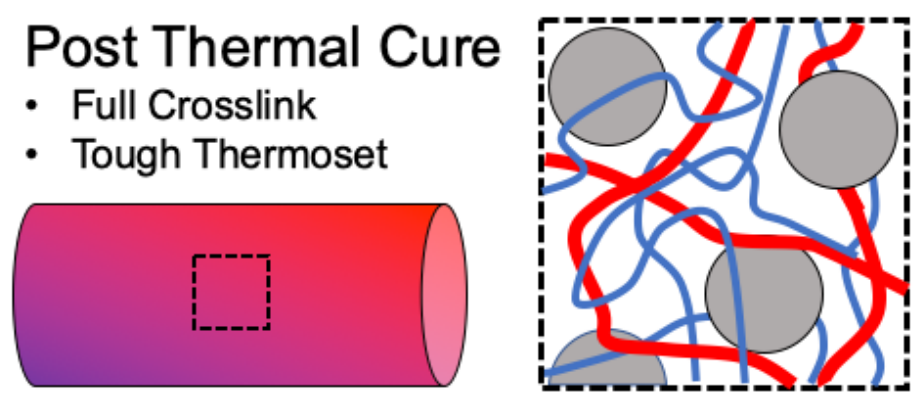
Scheme S2. Photograph of extrusion cartridge situated in printhead with connected UV LED oriented onto the print bed for in-line curing of printed filaments

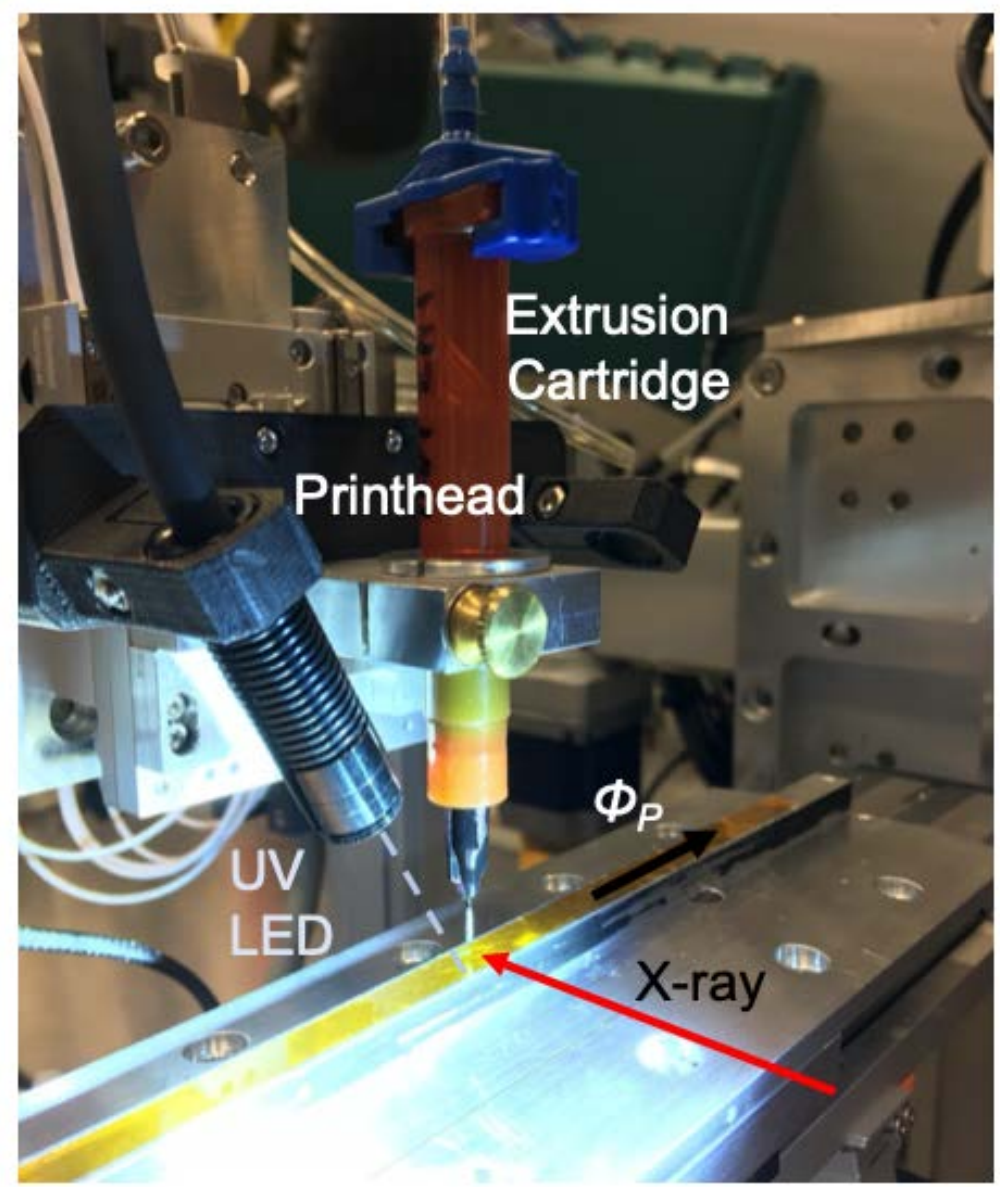

a)

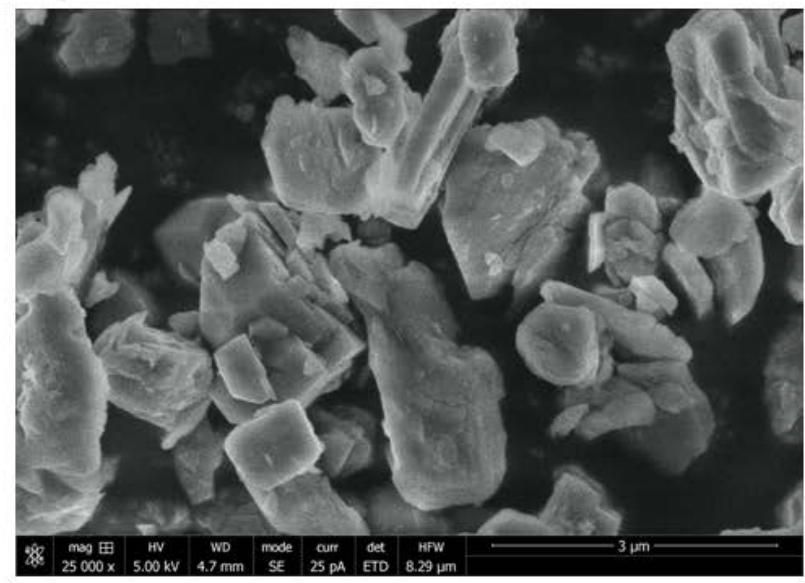

b)

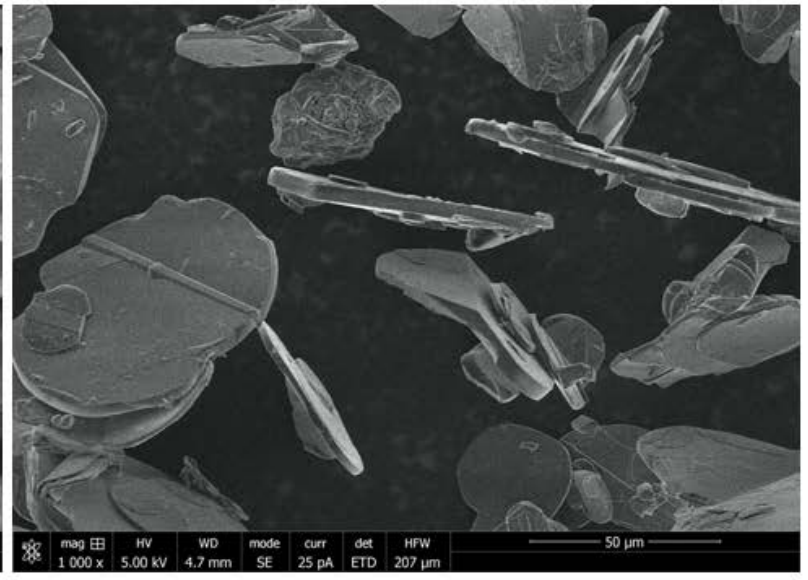

Figure S1. Scanning electron microscopy (SEM) of a) barium sulfate and b) boron nitride fillers. Scale bars are $3 \mu \mathrm{m}$ and $50 \mu \mathrm{m}$ respectively. 


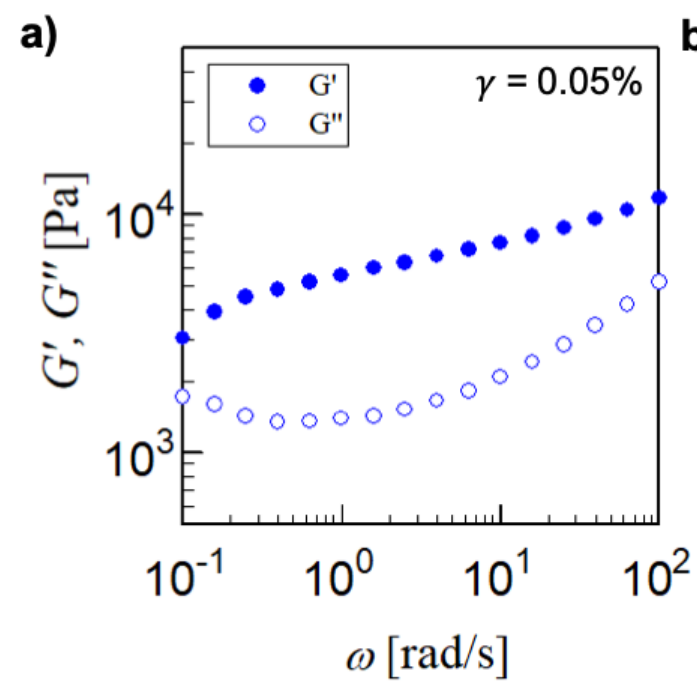

b)

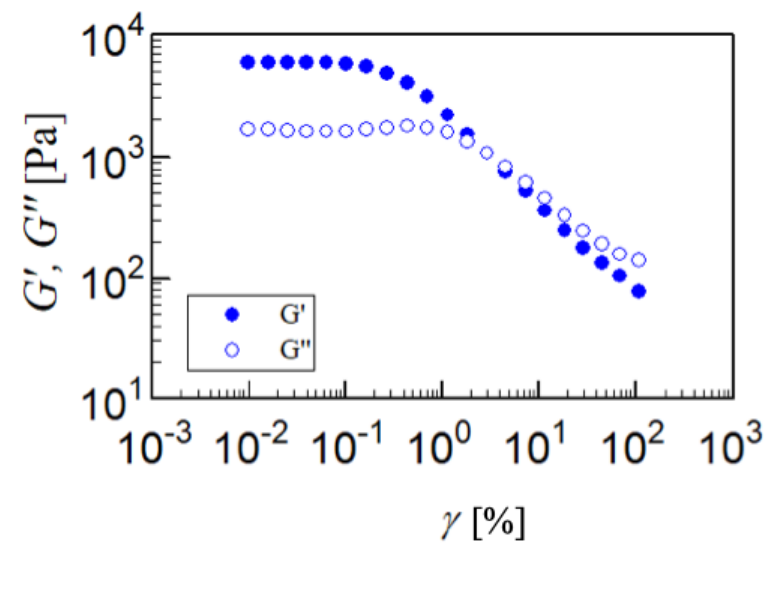

Figure S2. a) Linear viscoelasticity of uncured resin at room temperature $\left(T=25^{\circ} \mathrm{C}\right)$ presented as storage modulus $G^{\prime}(\omega)$ and loss modulus $G^{\prime \prime}(\omega)$ at a strain amplitude of $\gamma=0.05 \%$. $G^{\prime}(\omega)>$ $G$ " $(\omega)$ over the accessible frequency regime. Macroscopically, flow will be resisted if the structure is beyond the critical gel point, where the percolation of structure becomes sample spanning. At the rheological gel point, power-law scaling in the frequency dependence of the dynamic modulus $\left(G^{\prime}(\omega) \propto G^{\prime \prime}(\omega) \propto \omega^{\chi}\right)$ is observed. The static, linear viscoelasticity would suggest the resin is not a critical gel as $G^{\prime}(\omega)>G^{\prime \prime}(\omega)$ and power law scaling is not observed within the frequency window. b) Non-linear viscoelasticity of uncured resin at room temperature $\left(T=25{ }^{\circ} \mathrm{C}\right)$. Storage modulus $G^{\prime}(\omega)$ and loss modulus $G^{\prime \prime}(\omega)$ measured at a frequency of $\omega=1 \mathrm{rad} / \mathrm{s}$ at increasing strain amplitude (in percent strain). Linear viscoelastic regime extends to $\gamma \sim 0.2 \%$, while a cross over in $G$ ' and $G$ " is identified at $\gamma=3 \%$. 


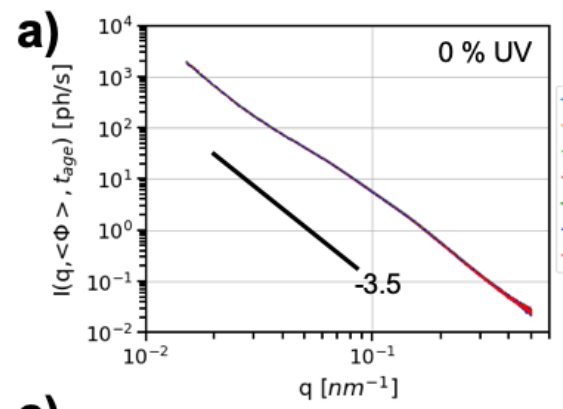

c)

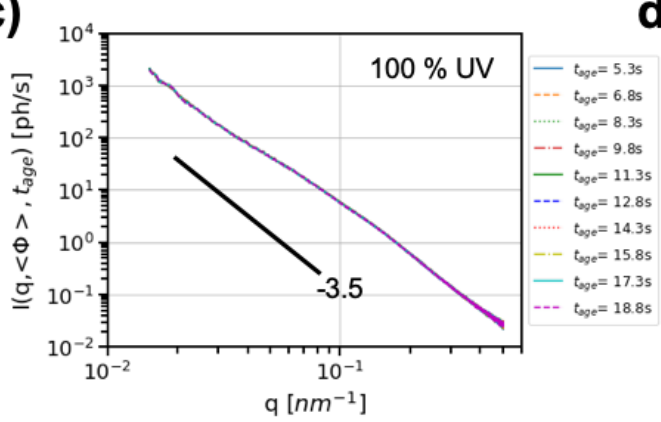

b)

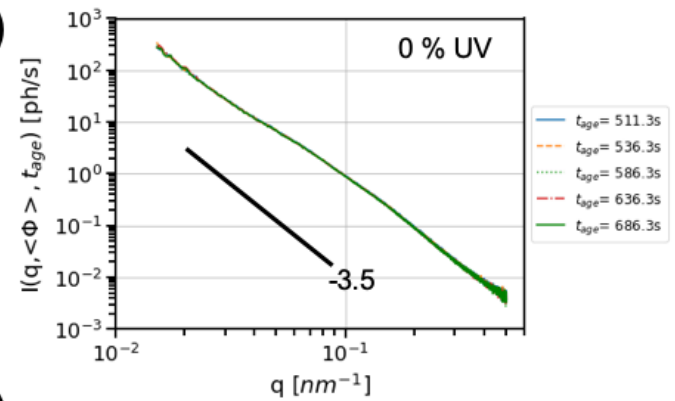

d)

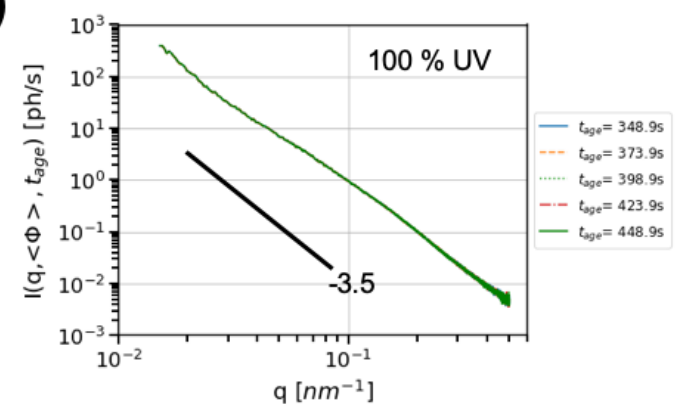

Figure S3. Time-resolved radially averaged small angle X-ray scattering (SAXS) spectra $I(q,<\phi>$, tage $)$ from time slices during in-operando printing experiments $\left(v_{x}=1 \mathrm{~mm} / \mathrm{s}\right)$ a) $0 \%$ UV in-situ printing at early tage and b) $0 \%$ UV several hundred seconds after filament deposition. c) 100\% UV in-situ printing at early tage d) 100\% several hundred seconds after filament deposition. SAXS spectra appears to be independent of tage in each sample and across the printing procedure.

a)

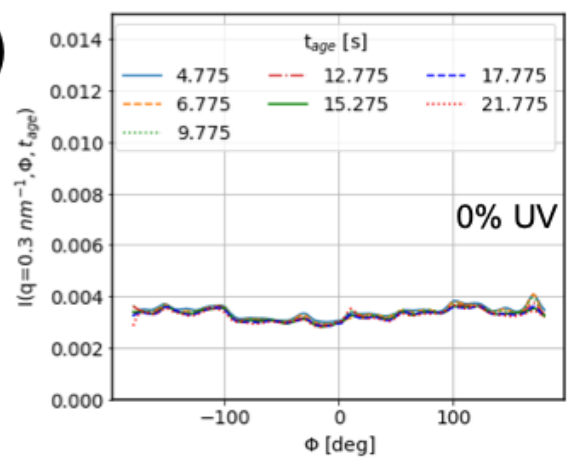

c)

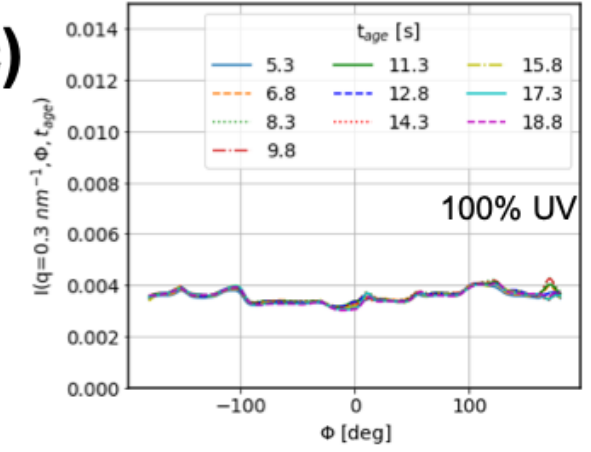

b)

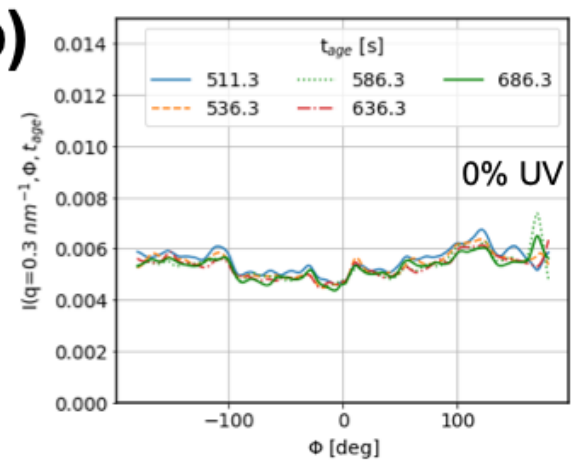

d)

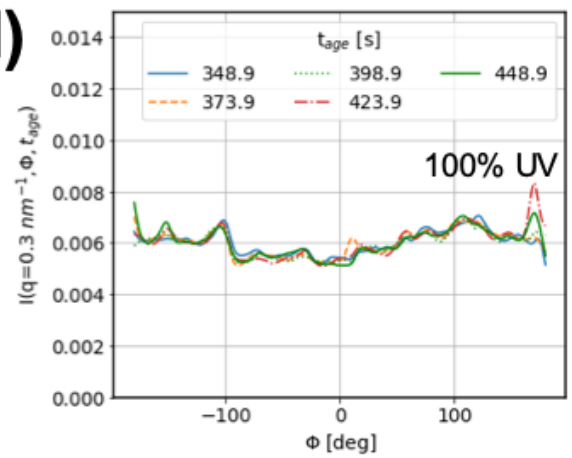

Figure S4. Azimuthal integration of time-resolved, small angle X-ray scattering (SAXS) spectra $\mathrm{I}\left(q=0.3 \mathrm{~nm}^{-1}, \phi\right.$, tage $)$ from equivalent time slices in Figure S3. $\phi$-dependence of all spectra are isotropic and appear to be independent of tage in each sample. 
a)

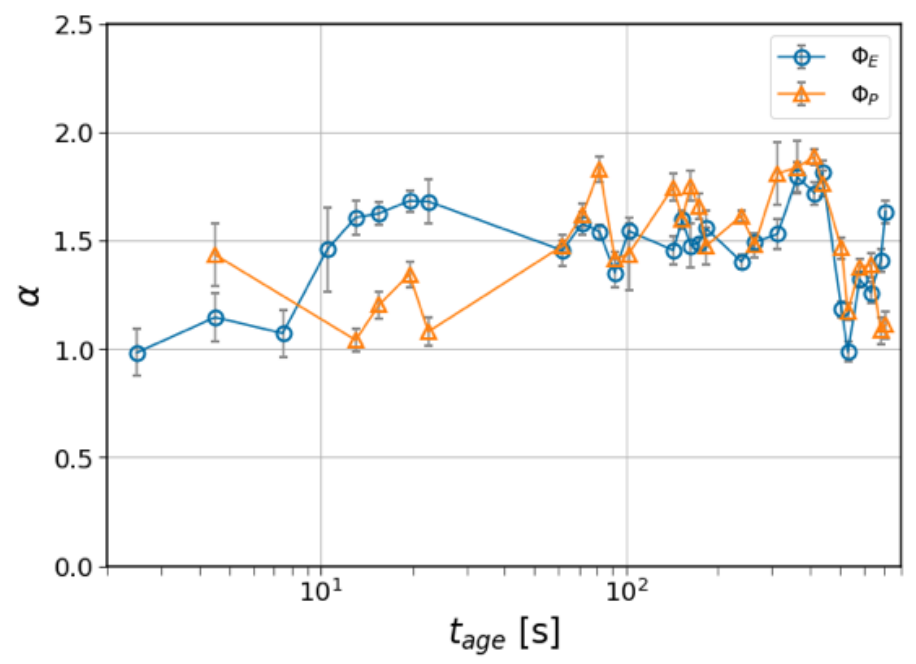

b)

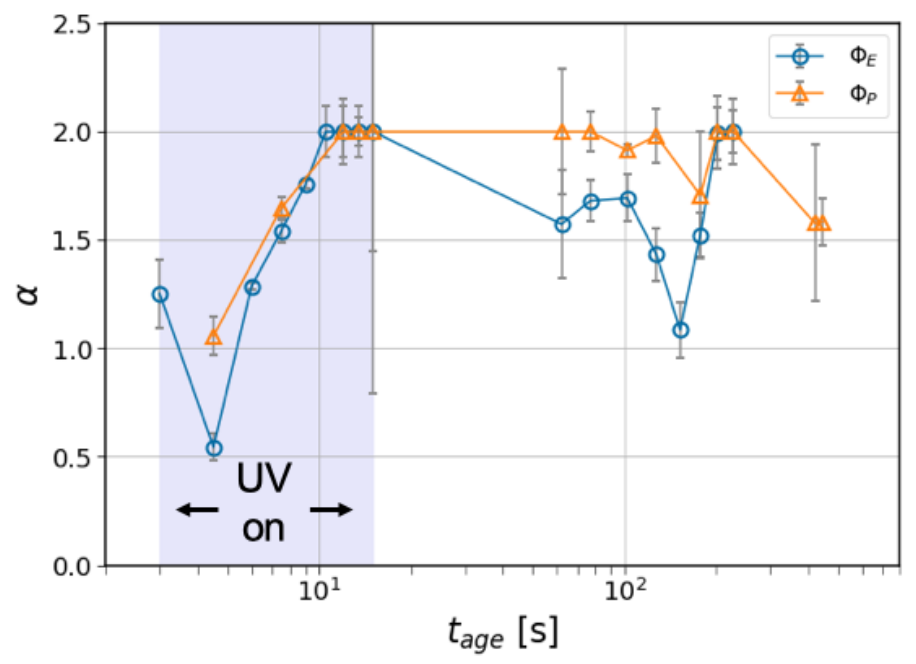

Figure S5. Directionally dependent trends in KWW stretching/compression exponent $\alpha$ for prints at $v_{x}=1 \mathrm{~mm} / \mathrm{s}$ and $10 \mathrm{psi}$ under a) $0 \% \mathrm{UV}$ and b) $100 \% \mathrm{UV} . \alpha$ is obtained from fits to $\mathrm{g}_{2}$ using eq. 3 in main text and plotted as function of tage at $q=0.029 \mathrm{~nm}^{-1}$. For $100 \%$ UV condition, the tage range of UV illumination is displayed in purple. 
a)

b)
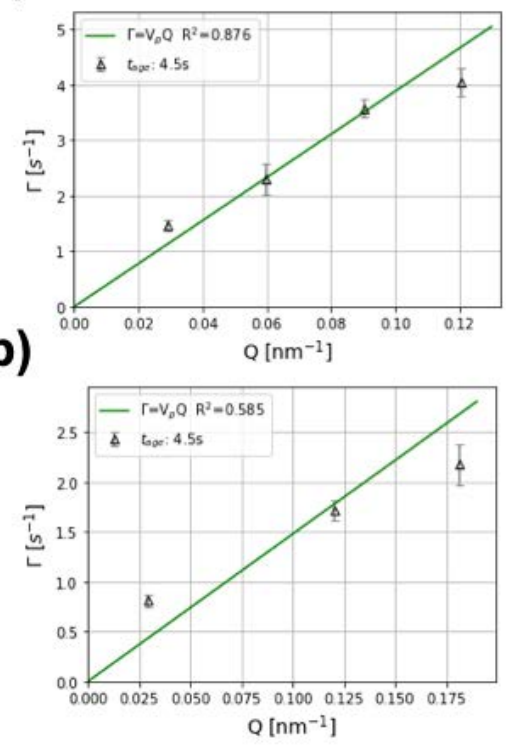
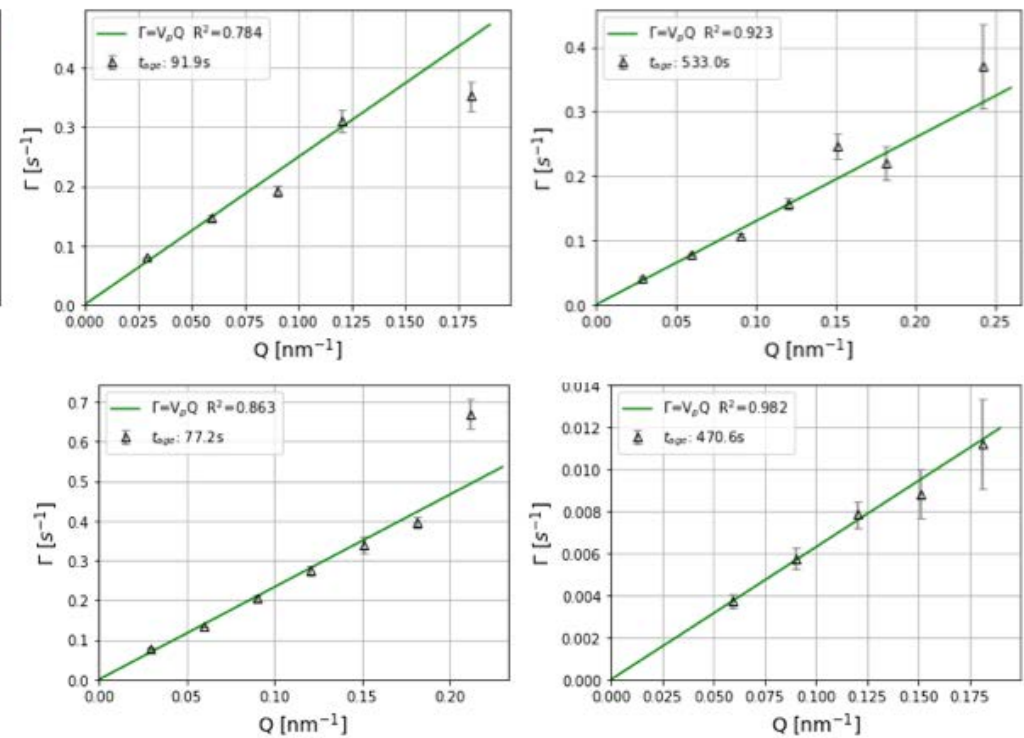

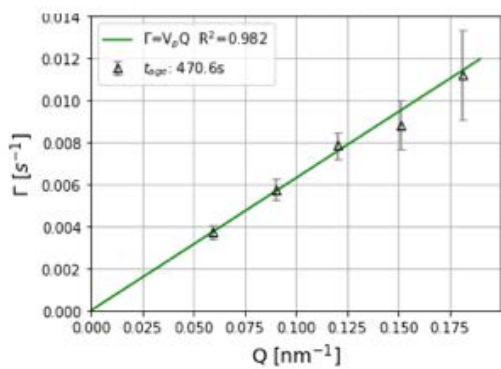

Figure S6. Relaxation rate $\Gamma$ scaling with $q$ and fits ballistic dynamics $\left(\Gamma=V_{p} q\right)$ where $V_{p}$ is the displacement velocity in $\mathrm{nm} / \mathrm{s}$ at representative tage for a) $0 \% \mathrm{UV}$ and b) $100 \% \mathrm{UV}$ at $v_{x}=1 \mathrm{~mm} / \mathrm{s}$ print speed and $P=10 \mathrm{psi}$ 
a)
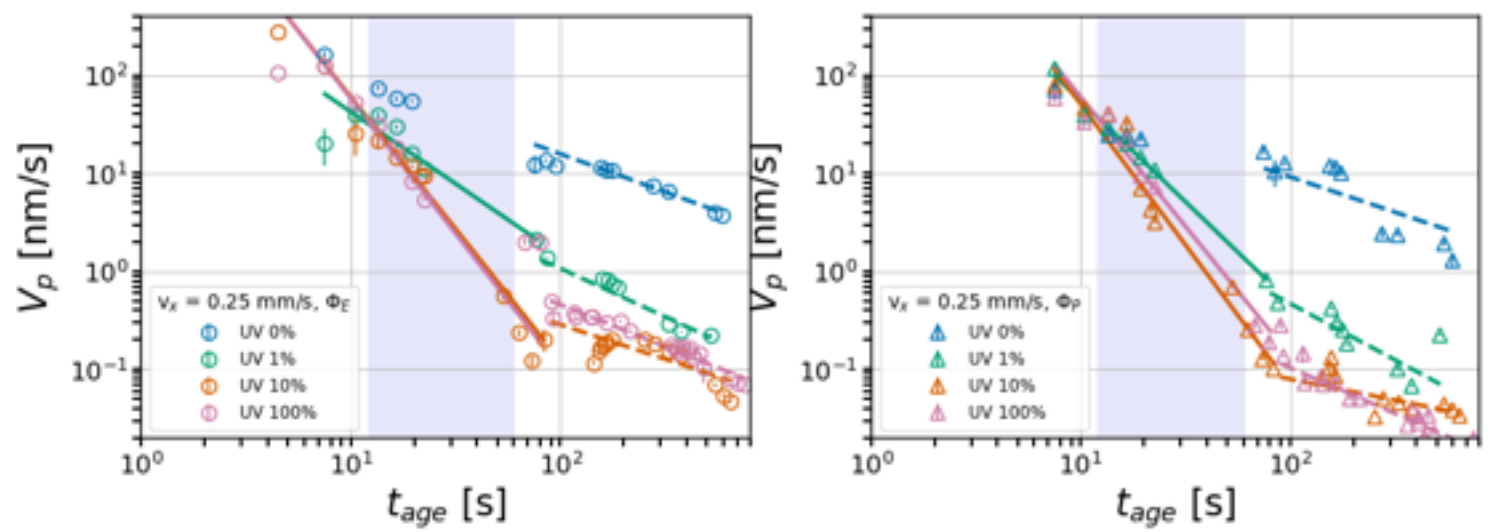

b)
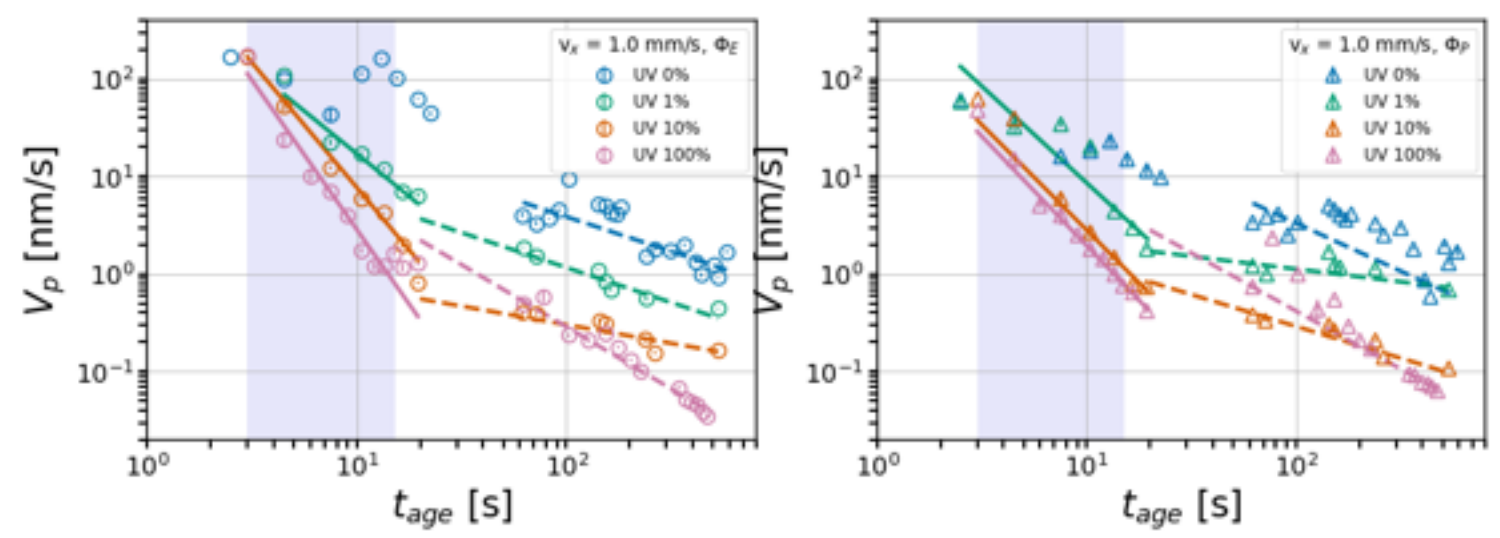

c)
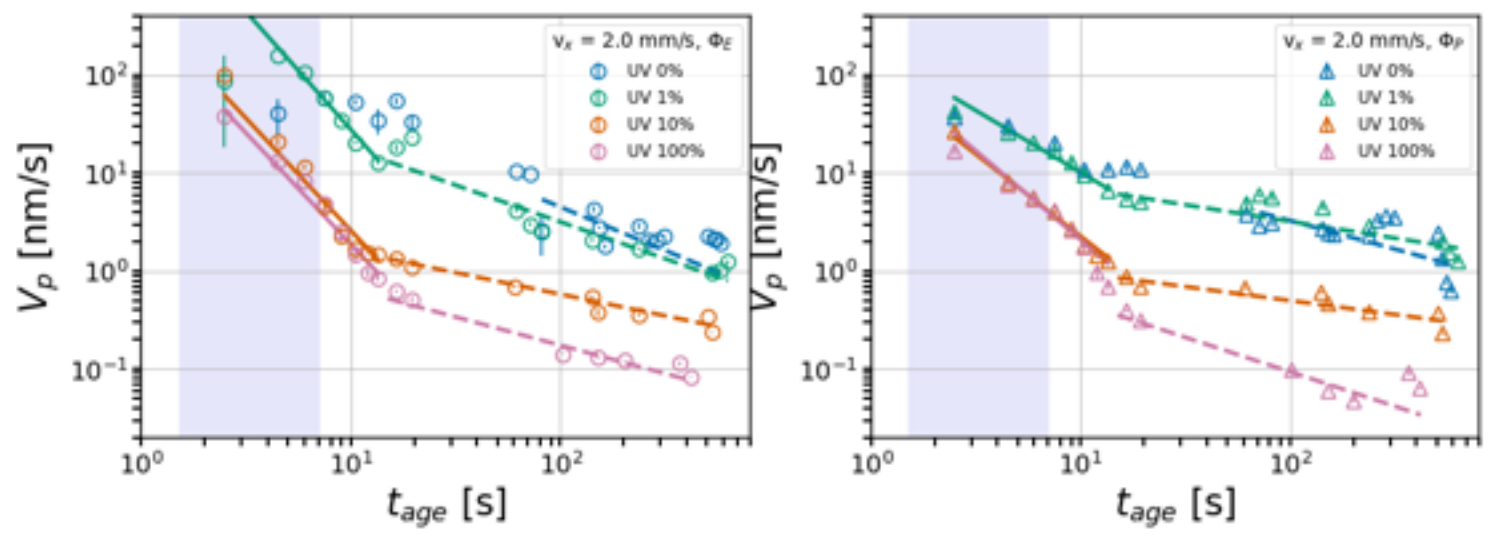

Figure S7. Compiled power law fits to evolution of $V_{p}$ with tage for printing at a) $v_{x}=0.25 \mathrm{~mm} / \mathrm{s}$ and $8 \mathrm{psi} \mathbf{b}) v_{x}=1 \mathrm{~mm} / \mathrm{s}$ and $10 \mathrm{psi}$ and c) $v_{x}=2 \mathrm{~mm} / \mathrm{s}$ and $15 \mathrm{psi}$ in the extrusion ( $\left.\phi_{\mathrm{E}}\right)$ and printing $(\phi \mathrm{p})$ directions. Time resolved dynamics follow scaling law $V_{p} \sim \operatorname{tage}^{m}$, where $m$ is the power law decay factor. In all cases at $0 \%$ UV radiation, a single power law is observed at late tage. Cutoff times between two power law regimes are a function of print speed where $t_{c}=90 \mathrm{~s}, 20 \mathrm{~s}, 15 \mathrm{~s}$ for $v_{x}=0.25,1$, and $2 \mathrm{~mm} / \mathrm{s}$ respectively. UV illumination duration is shaded in purple. 

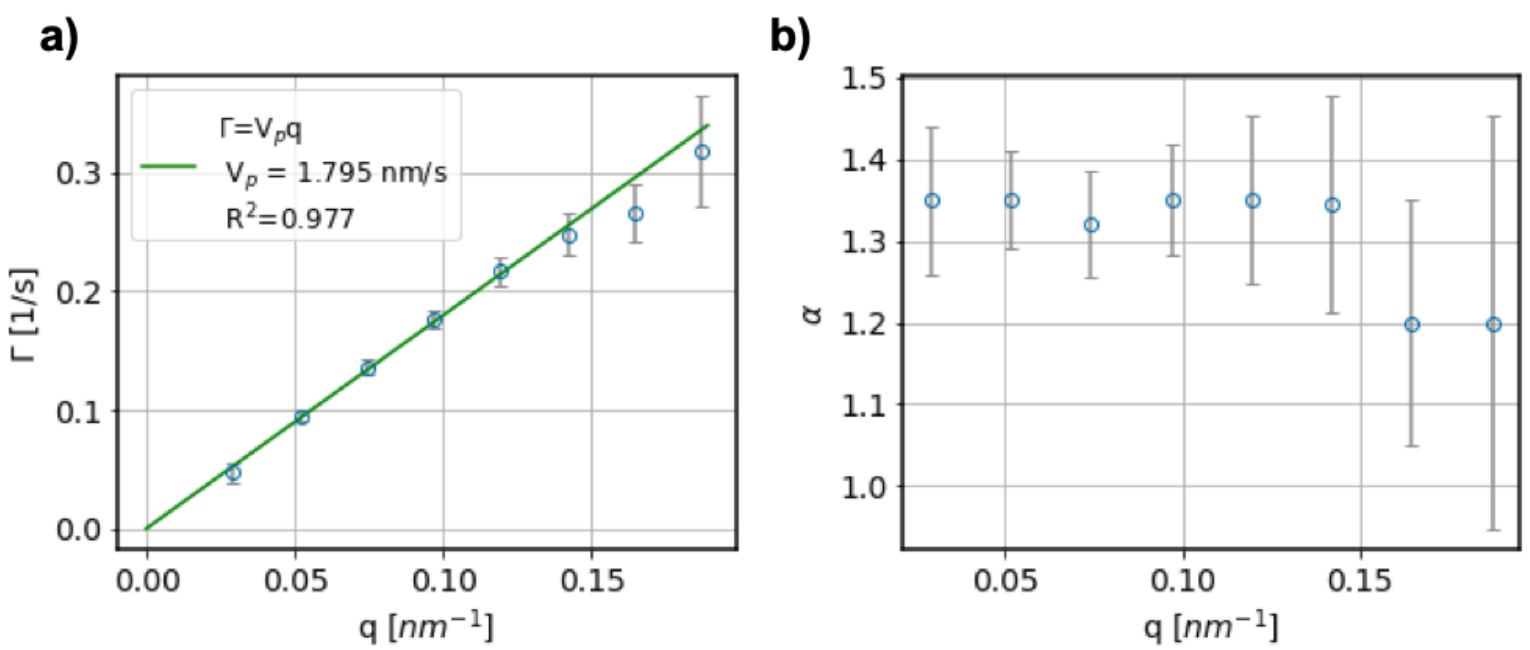

Figure S8. Equilibrium dynamics of uncured resin sample measured at room temperature in absence of external forces a) Relaxation rate $\Gamma\left(\mathrm{s}^{-1}\right)$ vs $q$-vector scaling reveals linear scaling with $V_{p}=1.795 \mathrm{~nm} / \mathrm{s}$. b) KWW exponent $\alpha$ indicates compressed exponential decays $(\alpha>1)$ across entire $q$ range. 
a)
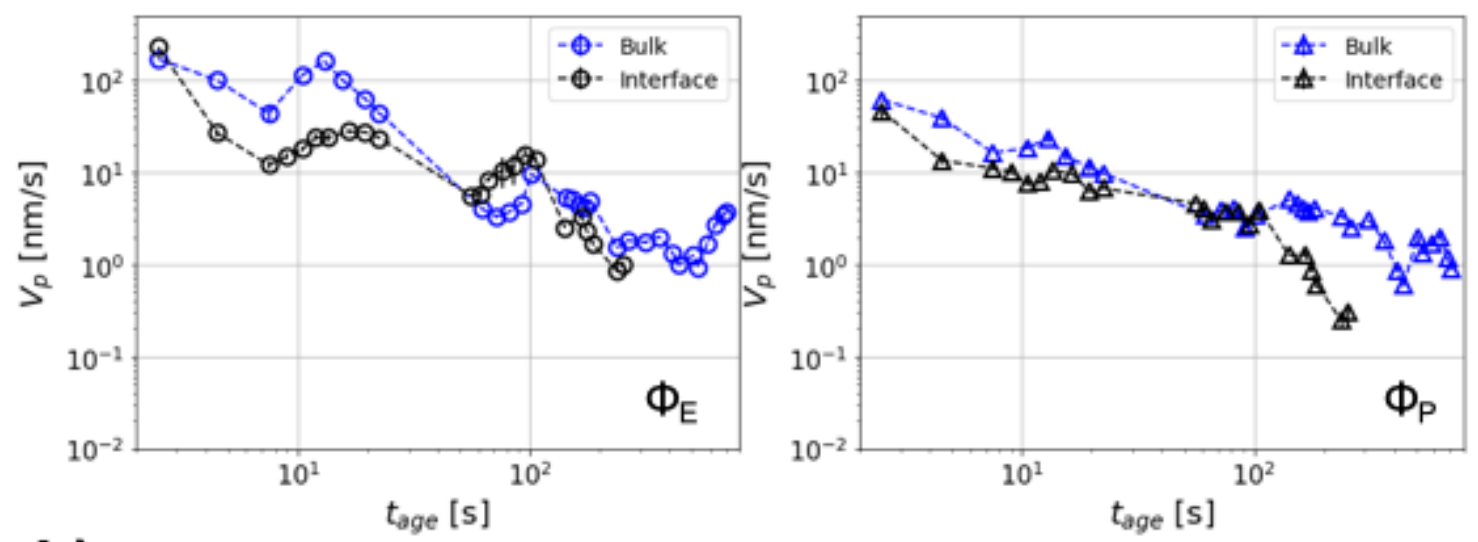

b)
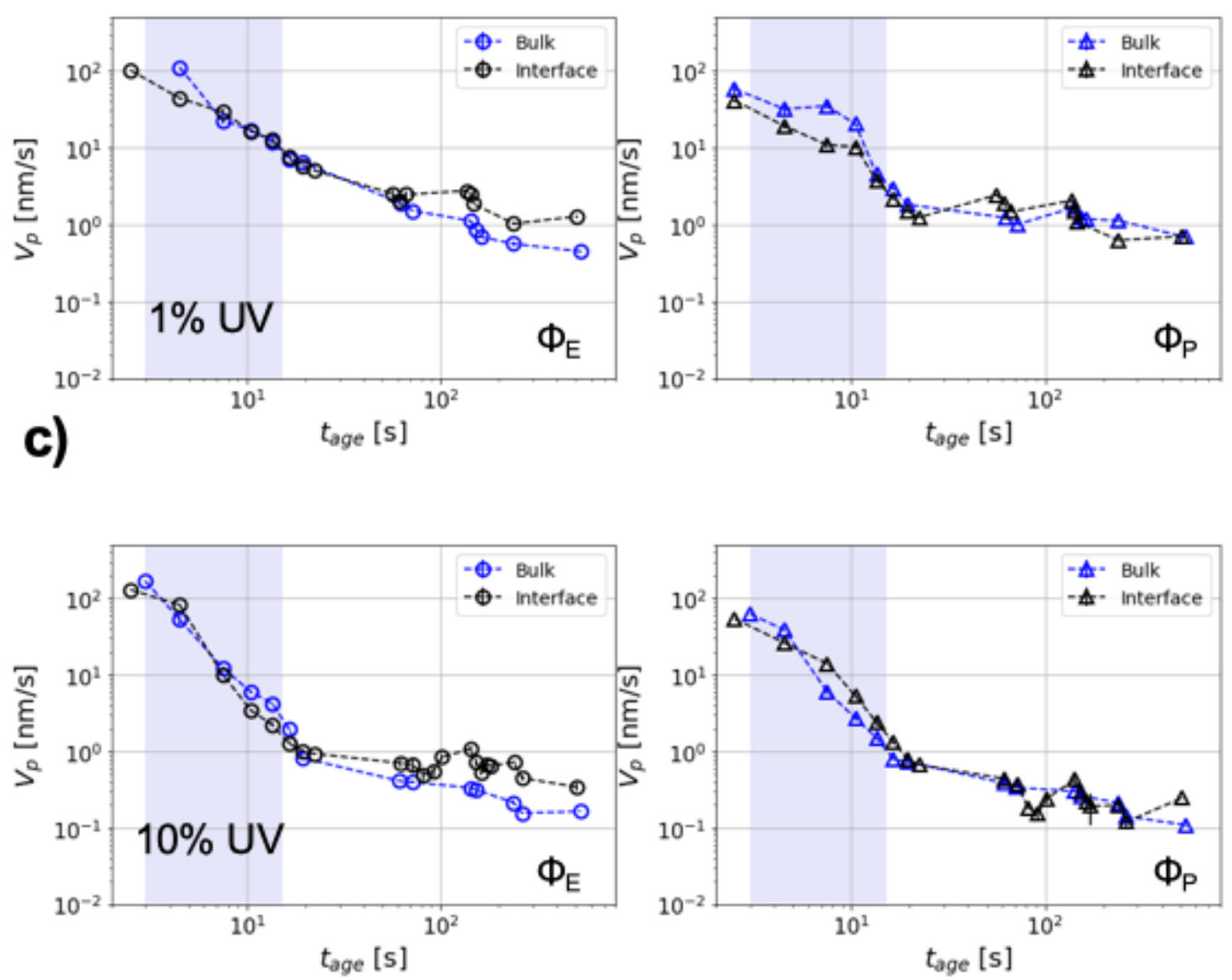

Figure S9. Additional spatially resolved dynamics comparing temporal evolution of $V_{p}$ at the filament interface, $h=550 \mu \mathrm{m}$ (black) and in the bulk filament $h=300 \mu \mathrm{m}$ (blue) at a) $0 \% \mathrm{UV}$, b) $1 \% \mathrm{UV}$ and c) $10 \% \mathrm{UV}$ at $v_{x}=1 \mathrm{~mm} / \mathrm{s}$ print speed and $10 \mathrm{psi}$. At $0 \% \mathrm{UV}$, the evolution of $V_{p}$ appears to be slightly slower at the interface across the observed tage range. The terminal dynamics of $V_{p}$ are comparable with the equilibrium dynamics (defined in Figure 6 of main text), especially in the $\phi_{E}$ direction. 
a)

$v_{x}=0.25 \mathrm{~mm} / \mathrm{s}$

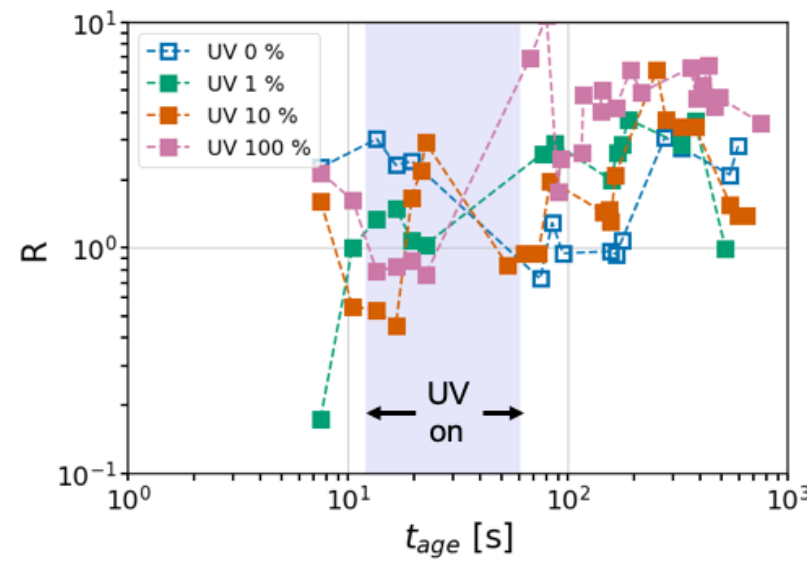

b) $\quad v_{x}=2 \mathrm{~mm} / \mathrm{s}$

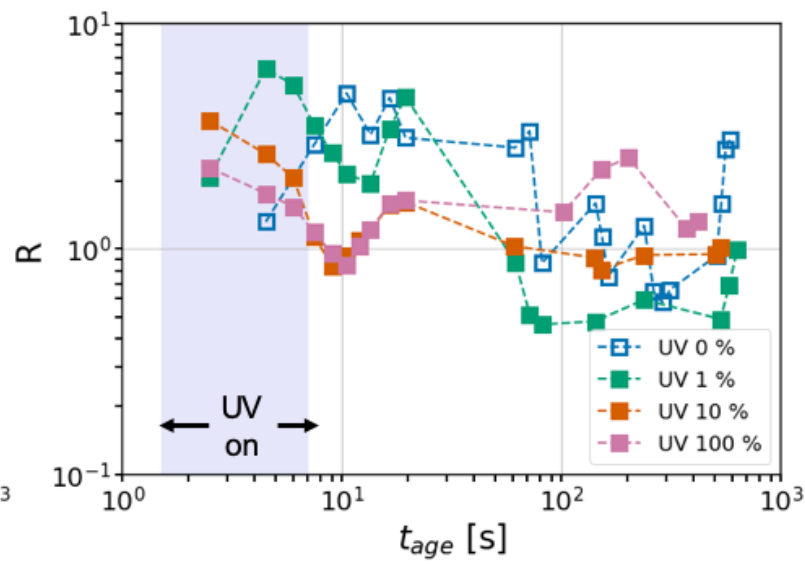

Figure S10. Trends in dynamic anisotropy factor $R$ with tage at printing speed a) $v_{x}=0.25 \mathrm{~mm} / \mathrm{s}$ and b) $v_{x}=2 \mathrm{~mm} / \mathrm{s}$ for UV conditions from $0 \%$ to $100 \%$
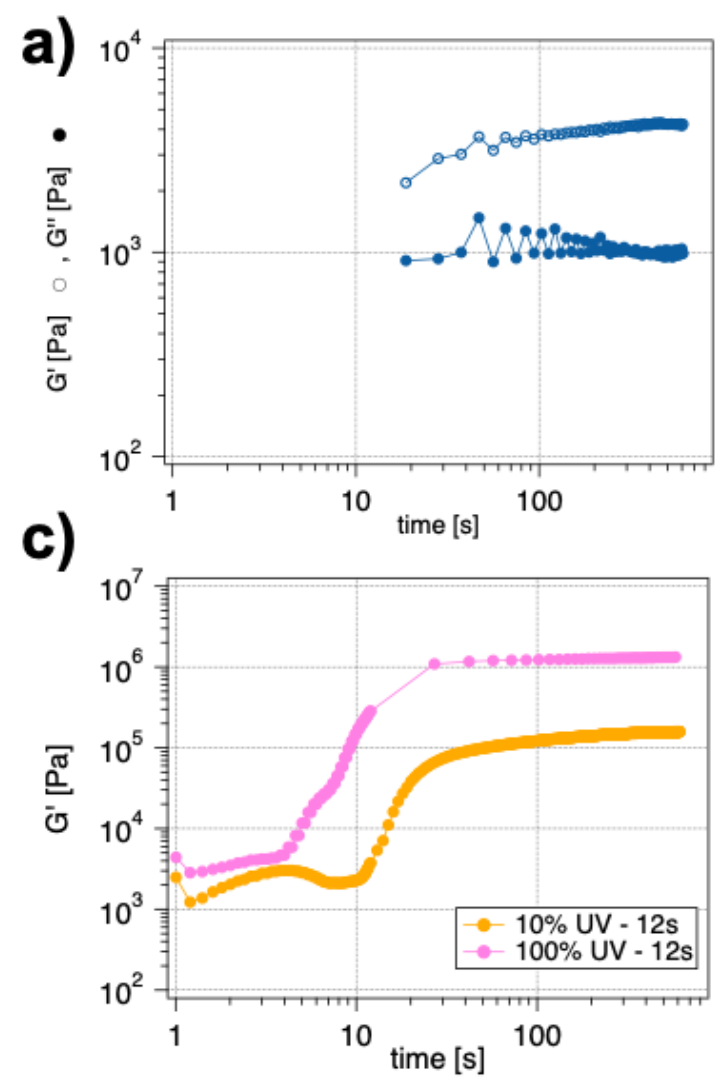
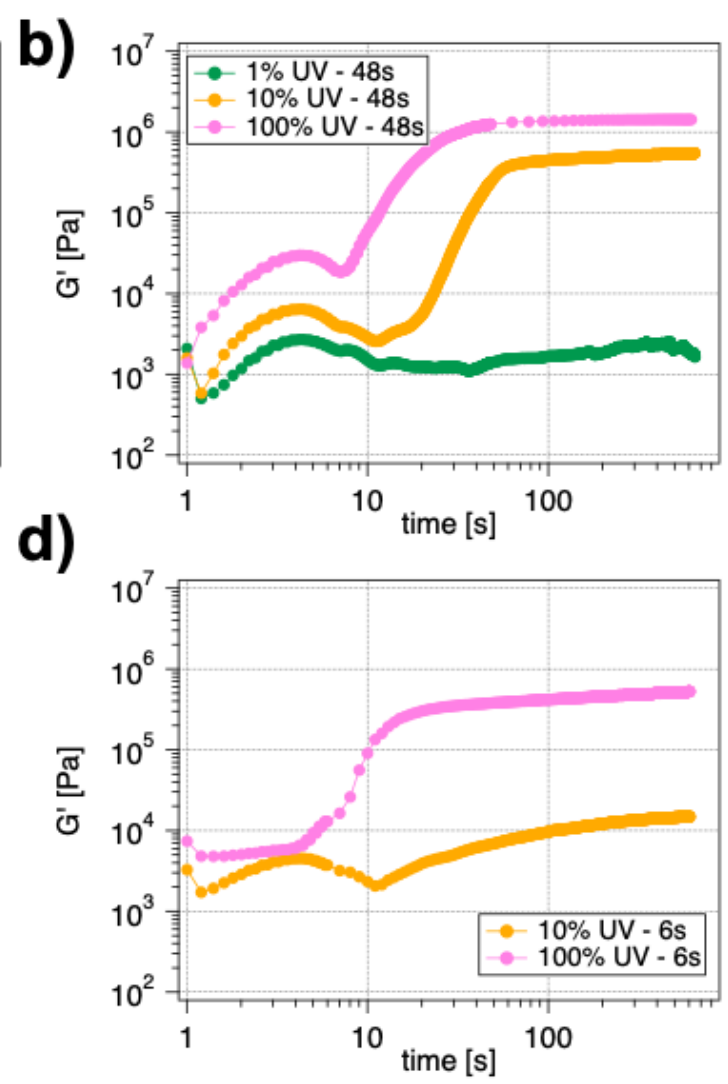

Figure S11. a) Development of storage modulus $G$ ' and loss modulus $G$ ” during structural recovery in uncured resin after $30 \mathrm{~s}$ of continuous shear at $\gamma^{*}=0.01 \mathrm{~s}^{-1}$. Viscoelasticity measured at single frequency $\omega=1 \mathrm{rad} / \mathrm{s}$ and constant strain amplitude $\gamma=0.05 \%$. Growth of $G$ ' during insitu UV shear rheology for various UV intensities and duration from time $=0 \mathrm{~s}$ : b) $48 \mathrm{~s} \mathrm{c)} 12 \mathrm{~s}$ and d) $6 \mathrm{~s}$ corresponding to XPCS results at $v_{x}=0.25 \mathrm{~mm} / \mathrm{s}, 1 \mathrm{~mm} / \mathrm{s}$, and $2 \mathrm{~mm} / \mathrm{s}$ respectively. $1 \% \mathrm{UV}$ 
for $6 \mathrm{~s}$ and $12 \mathrm{~s}$ was not sufficient to fully cure the sample in the rheometer geometry and therefore was not plotted.

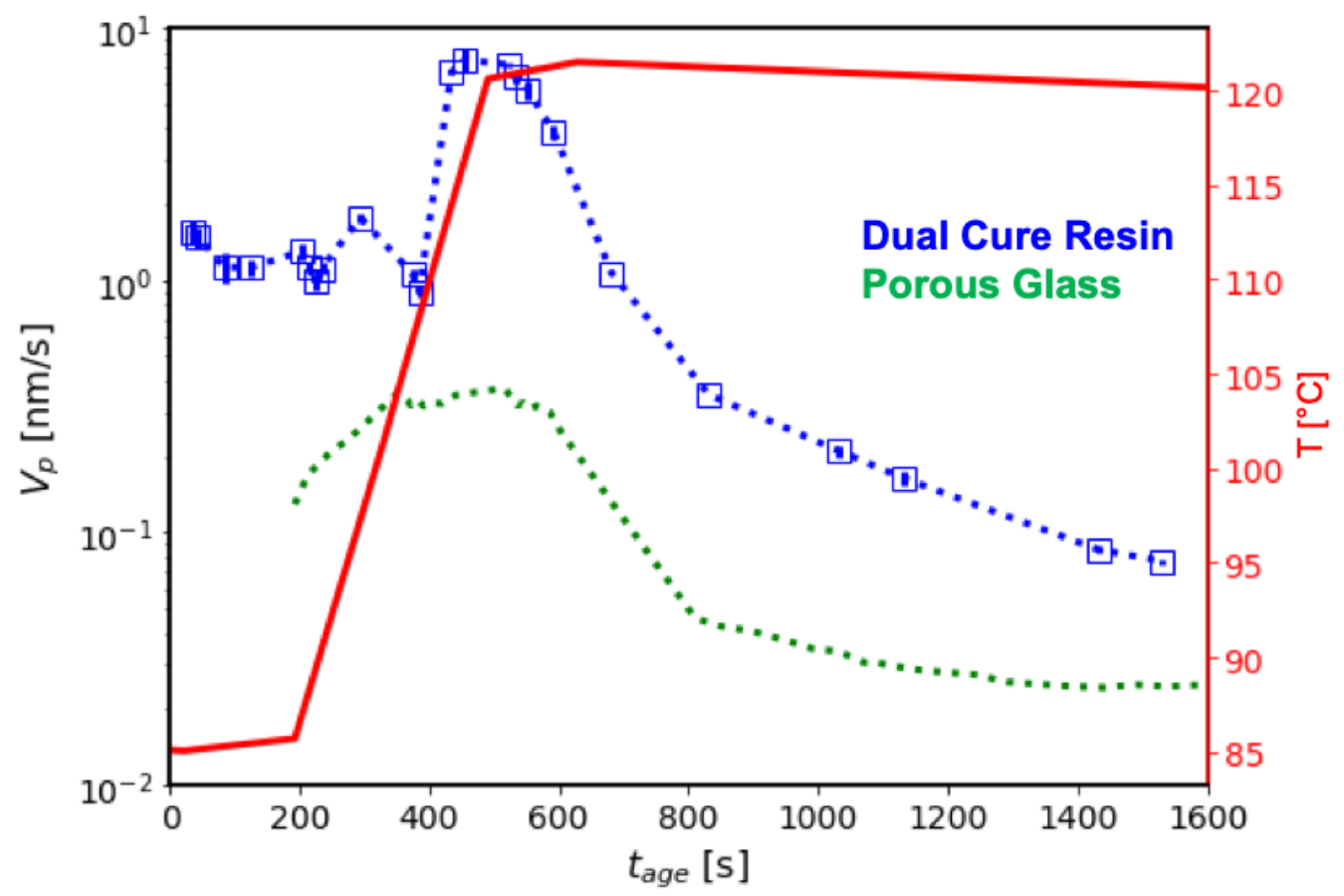

Figure S12. Evolution of nanoscale dynamics expressed by the local displacement velocity $\left(V_{p}=\right.$ $\Gamma / q$ ) over the full thermal curing procedure of dual cure resin. Sample is heated from preheat state at $T=85^{\circ} \mathrm{C}$ through to $T_{\text {cure }}=120^{\circ} \mathrm{C}$ at a heating rate of $5{ }^{\circ} \mathrm{C} / \mathrm{min}$. Temperature is plotted on the secondary y axis. Dynamics are compared to that for static porous glass sample (in green)

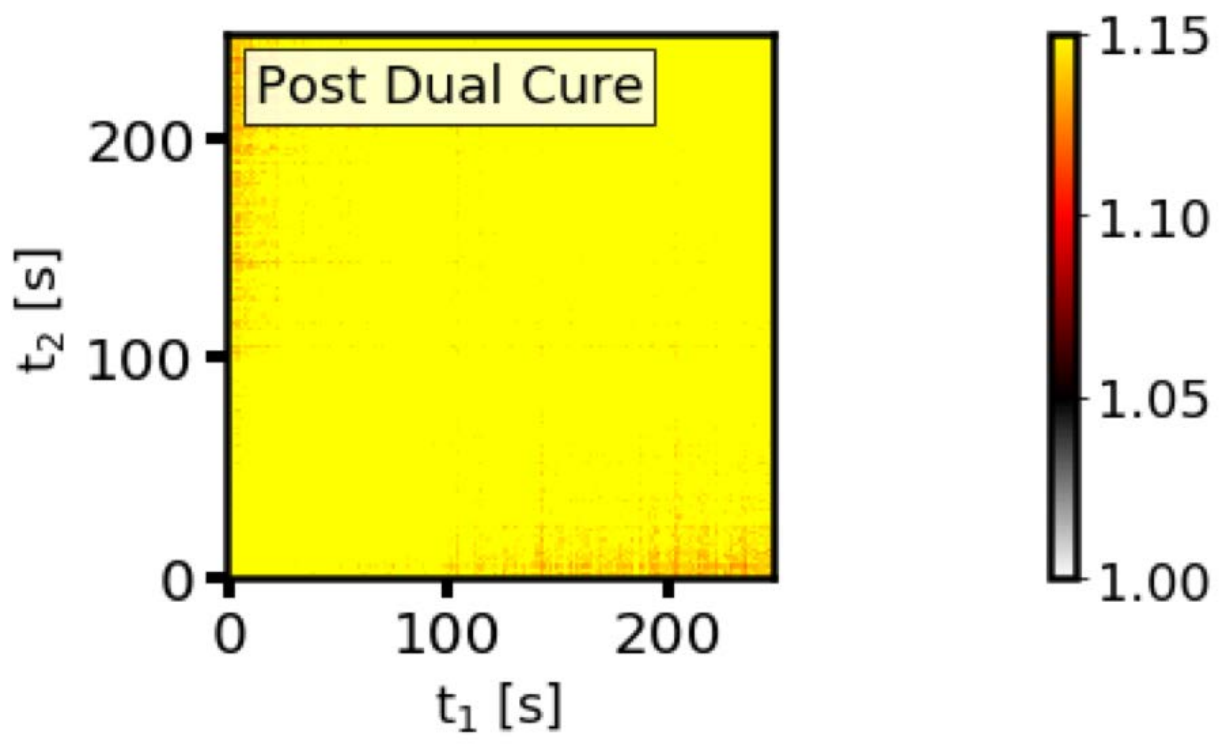


Figure S13. Two-time correlation function $\left(q=0.029 \mathrm{~nm}^{-1}\right)$ of fully cured resin after in-situ UV curing $(100 \%)$ and ex-situ thermal curing $\left(T_{\text {cure }}=120^{\circ} \mathrm{C}\right)$. Dynamics extend to significantly longer time scales compared to the uncured state and each individual curing step (UV or thermal) 